\title{
Evaluation of Combined Disc method for the detection of metallo- $\beta$-lactamase producing Gram negative bacilli
}

\author{
Maria Omair, Javaid Usman, Fatima Kaleem, Afreenish Hassan, Ali Khalid and Qanita Fahim \\ Department of Microbiology, Army Medical College, National University of Sciences and Technology, Abid Majeed Road, \\ Rawalpindi, Pakistan. \\ E-mail: maria2283@gmail.com
}

Received 29 June 2011; received in revised form 27 September 2011; accepted 23 October 2011

\begin{abstract}
Aims: Infections due to metallo- $\beta$-lactamase (MBL) producing Gram negative rods are a cause of high mortality and morbidity. Early detection by an economical and accurate method may improve patient outcome. This study was aimed to evaluate the diagnostic accuracy of combined disc method for MBL detection by comparing it with MBL-Etest.

Methodology and Results: This cross-sectional, validation study was carried out in the Department of Microbiology, Army Medical College, National University of Sciences and Technology, Rawalpindi, over a period of six months. A total of 52 non-duplicate Gram-negative rods isolated from the routine clinical specimens and found resistant to meropenem/imipenem on Kirby Bauer Disc Diffusion method were subjected to two tests for metallo- $\beta$-lactamase detection. One was combined Disc test using imipenem with Ethylene Diamine Tetraacetic Acid (EDTA), where a strain showing an increase in zone of inhibition of combined disc of $\geq 7 \mathrm{~mm}$ as compared to imipenem alone, was considered as MBL producer and the other one was MBL-Etest for which results were interpreted as per manufacturer's guidelines. Combined disc method for MBL detection was found to have a sensitivity, specificity, positive predictive value, negative predictive value and accuracy of $97.5 \%, 100 \%, 100 \%, 92 \%$ and $98 \%$.

Conclusion, Significance and Impact of study: Combined disc method is an economical and reliable method for metallo- $\beta$-lactamase detection which can be used routinely in any laboratory.
\end{abstract}

Keywords: Combined Disc test, MBL-Etest, Metallo- $\beta$-lactamase (no italicize)

\section{INTRODUCTION}

Carbapenem group of antibiotics play a vital role in the management of hospital-acquired Gram negative infections, because of their broad spectrum activity and stability against hydrolysis by most of the $\beta$-lactamases, including extended spectrum $\beta$-lactamases (ESBLs) (Irfan et al., 2008). Carbapenem hydrolyzing enzymes can be divided into two main sub types; Serine $\beta$-lactamases (Ambler class $A$ and $D$ ) and metallo- $\beta$-lactamases (Ambler class B) (Marchiaro et al., 2005; Louis et al., 2007; Queenan and Bush, 2007). The emergence of MBLs in Gram negative bacilli is becoming a therapeutic challenge as these enzymes render all penicillins, cephalosporins and carbapenems ineffective (Louis et al., 2007, Irfan et al., 2008).

MBL was reported for the first time in 1991 in Japan and since then nosocomial outbreaks due to metallo- $\beta$ lactamase (MBL) producing Gram negative bacilli are being increasingly reported from different parts of the world (Irfan et al., 2008; Kaleem et al., 2010). Limited, expensive and toxic treatment options as well as high risk of spread to other Gram negative bacilli via plasmidmediated mechanism is a matter of great concern; therefore rapid detection of $\mathrm{MBL}$ production is essential to modify therapy and to prevent their dissemination (Irfan et al., 2008).

Currently, no standardized method for MBL detection has been proposed. Polymerase Chain Reaction (PCR) is highly accurate and reliable but its accessibility is often limited to reference laboratories (Behera et al., 2008). Several non molecular techniques have been studied, all taking advantage of the fact that MBLs require zinc or another heavy metal for their action and their activities are inhibited by chelating agents e.g. EDTA, dipicolinic acid and thiol compounds (Arakawa et al., 2000; Berges et al., 2007; Behera et al., 2008; Shobha et al., 2009). Various phenotypic methods for MBL detection are combined disc method, double disc synergy method and Etest. MBLEtest is considered the phenotypic standard method for MBL detection but the test is expensive (Monoharan et al., 2010). Double disc synergy and combined disc tests are economical and simple to perform but double disc test is observer dependent while CD test is measureable with lesser chances of subjective error (Monoharan et al., 2010).

In case of Pseudomonas, EDTA may produce a zone of inhibition due to its effect on the permeabilization of the drug through the outer membrane proteins (Ratkai et al., 2009). Therefore while detecting MBL using EDTA, a simultaneous determination of zone of inhibition of EDTA

\section{${ }^{*}$ Corresponding author}


alone, is mandatory to rule out false $\mathrm{MBL}$ detection (Ratkai et al., 2009).

The aim of the present study was to evaluate the combined disc method for MBL detection, in order to select a reliable and economical method for future MBL detection in Gram negative bacilli.

\section{MATERIALS AND METHODS}

\section{Sample Processing}

This Cross-sectional, validation study was carried out in Department of Microbiology, Army Medical College, National University of Sciences and Technology, from January 2010 to June 2010. A total of 52 non-duplicate isolates of the Gram negative bacilli, resistant to carbapenem on the Kirby Bauer disc diffusion method were included in the study by non-probability, convenient sampling. Routine clinical specimens like blood, urine, wound discharge (pus), high vaginal swab (HVS), sputum and nasobronchial lavage (NBL) received in the Department of Microbiology, Army Medical College, National University of Sciences and Technology, Rawalpindi were applied on appropriate culture media (Oxoid U.K.) and the pathogens were identified to species level by standard microbiological methods like Gram staining, colony morphology and analytical profile index (API) 20E. Resistance to carbapenems was determined by using standard Kirby Bauer disc diffusion method, according to Clinical and Laboratory Standards Institute (CLSI) guidelines using imipenem/meropenem $(10 \mu \mathrm{g})$ disc (Clinical and Laboratory Standards Institute, 2009). All the isolates showing zone of inhibition of $<16 \mathrm{~mm}$ for imipenem/meropenem were taken as resistant (Clinical and Laboratory Standards Institute 2009). The isolates of Gram negative bacilli which were resistant to imipenem/meropenem were further subjected to MBLEtest (taken gold standard method, for MBL detection) and imipenem (IPM)-EDTA combined disc Test.

\section{Imipenem (IPM)-EDTA combined disc Test}

The IPM-EDTA combined disk test was performed as described by Yong et al. (2002). Test organisms were inoculated on plates with Mueller Hinton agar (MHA) as recommended by the CLSI (Clinical and Laboratory Standards Institute, 2009). Two $10 \mu \mathrm{g}$ imipenem disks (Becton Dickinson) were placed on the plate, and $10 \mu \mathrm{L}$ of EDTA solution was added to one of them to obtain the concentration of $750 \mu \mathrm{g}$ of EDTA. For Pseudomonas aeruginosa isolates $(n=10)$, an extra blank disc was also applied on the plate to which $10 \mu \mathrm{L}$ of EDTA solution of the same concentration was added. The inhibition zones of the imipenem and imipenem-EDTA disks were compared after 16 to 18 hours of incubation at $35^{\circ} \mathrm{C}$. In the combined disc test, if the increase in inhibition zone of an isolate, with the imipenem and EDTA disc was $\geq 7 \mathrm{~mm}$ as compared to the imipenem disc alone, the isolate was considered as MBL positive (Berges et al., 2007; Behera et al., 2008). The zones of inhibition around EDTA were also recorded separately to later compare it to the result of $C D$ test (difference between the imipenem and imipenemEDTA inhibition zones), in order to rule out false MBL positivity.

\section{MBL-Etest}

A 0.5 McFarland's suspension of the each isolate was made separately and inoculated on a plate of MHA. The Etest MBL strip containing an antibiotic concentration gradient for imipenem (IP) alone of 4 to $256 \mu \mathrm{g} / \mathrm{mL}$ and imipenem in combination with a fixed concentration of EDTA (IPI) gradient of 1 to $64 \mu \mathrm{g} / \mathrm{mL}$ was applied on the plate. The plate was then incubated at $37{ }^{\circ} \mathrm{C}$ for $24 \mathrm{~h}$ according to the manufacturer's instructions (Etest $\mathrm{MBL}$ ). MIC ratio of IP (Imipenem)/IPI (Imipenem-EDTA) of $>8$ or $>3 \log _{2}$ dilutions, a phantom zone or a synergistic zone indicated MBL production (Figure 1) (Behera et al., 2008, Etest MBL).

\section{Statistical Analysis}

Data was analyzed using Statistical Package for the Social Sciences (SPSS) version-17. Descriptive statistics were used to describe the data. Sensitivity, specificity, positive predictive value (PPV), negative predictive value (NPV) and accuracy was calculated for combined disc method using Etest as standard method and utilizing the following $2 \times 2$ table.

\begin{tabular}{c|c|c|}
\multicolumn{2}{c}{ Etest } \\
\cline { 2 - 3 } CD Test & $\begin{array}{r}\text { True positives } \\
\text { (a) }\end{array}$ & False Positives \\
\cline { 2 - 3 } & False Negatives & True Negatives \\
(c) & (d) \\
\hline
\end{tabular}

$\begin{array}{ll}\text { Sensitivity } & = \\ \text { Specificity } & =d \times 100 / a+c \\ \text { PPV } & =a \times 100 / b+d \\ \text { NPV } & =d \times 100 / c+d \\ \text { Accuracy } & =(a+d) \times 100 / a+b+c+d\end{array}$




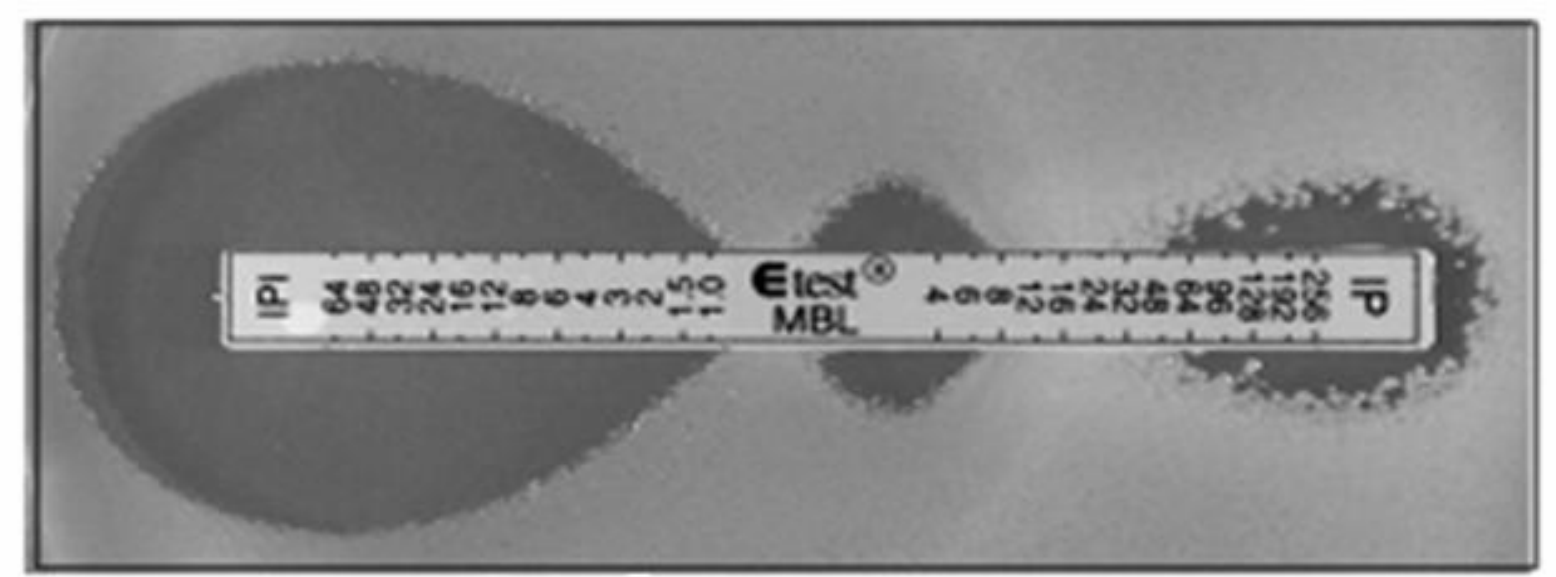

Figure 1: Phantom zone between IP and IPI (Imipenem +EDTA), indicative of MBL.

\section{RESULTS}

A total of 52 isolates of Gram negative bacilli resistant to imipenem/meropenem on Kirby Bauer disc diffusion method were included in the study. Out of these 52,23 $(44 \%)$ were from nasobronchial lavage (NBL), 10(19\%) were from pus, $6(11 \%)$ from urine, $5(9.6 \%)$ from catheter tips, $3(6 \%)$ from blood, $2(4 \%)$ from sputum, $2(4 \%)$ from ear swabs and only $1(2 \%)$ from high vaginal swab (HVS) (Table 1). Among these 52 isolates, 33 (63\%) were Acinetobacter spp., 10(19\%) were Pseudomonas spp. and 9(17\%) were Escherichia coli.

Table 1: Representation of $\mathrm{MBL}$ production in various Clinical specimens.

\begin{tabular}{lll}
\hline $\begin{array}{l}\text { Clinical } \\
\text { specimens }\end{array}$ & $\begin{array}{l}\text { Number of isolates } \\
\text { containing MBL } \\
\text { producing GNRs }\end{array}$ & Percentages \\
\hline $\begin{array}{l}\text { Nasobronchial } \\
\text { lavage }\end{array}$ & $23 / 52$ & $44 \%$ \\
Pus & $10 / 52$ & $19 \%$ \\
Urine & $6 / 52$ & $12 \%$ \\
Catheter Tips & $5 / 52$ & $9.6 \%$ \\
Blood & $3 / 52$ & $6 \%$ \\
Sputum & $2 / 52$ & $4 \%$ \\
Ear Swab & $2 / 52$ & $4 \%$ \\
High Vaginal & $1 / 52$ & $2 \%$ \\
Swab & & \\
\hline
\end{tabular}

Out of a total of 52 clinical isolates, $40(77 \%)$ came out to be positive by both CD Test and Etest (Figures 1 and 2 ). While $11(21 \%)$ were negative for MBL production as detected by both the methods and there was only one isolate $(1.9 \%)$ which was positive for MBL production by Etest but negative by CD Test. Using the $2 \times 2$ table, the sensitivity, specificity, positive predictive value, negative predictive value and accuracy of CD test were found to be $97.5 \%, 100 \%, 100 \%, 92 \%$ and $98 \%$, respectively.

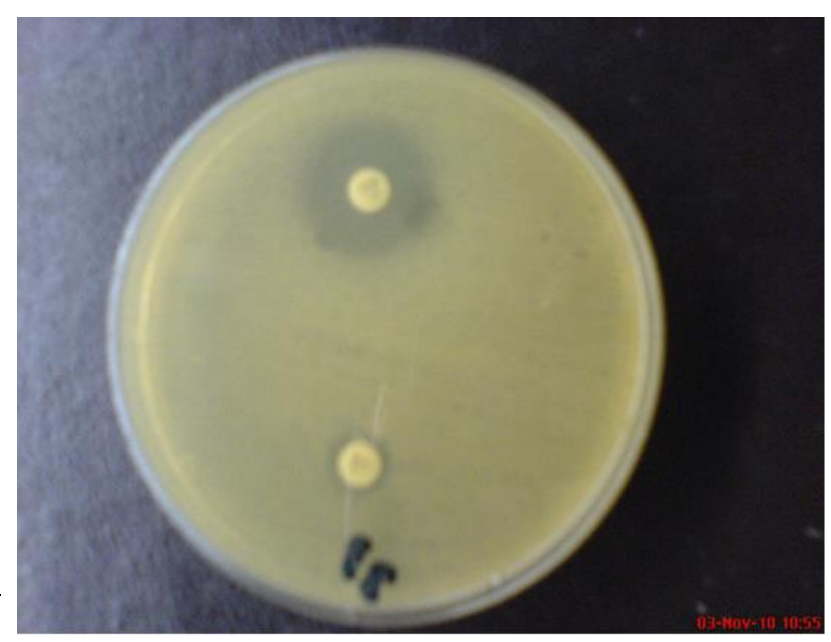

Figure 2: An isolate of MBL producing Gram negative bacillus showing positive Combined disc test.

Comparison of the inhibition zones of EDTA and the difference between the inhibition zones of imipenem and imipenem-EDTA revealed a t-value of 3.1 and $p$-value of 0.012 indicating that the difference is significant so in the current isolates of Pseudomonas, the EDTA is actually detecting MBL and its not merely due to increase in the permeability of the drug (Table 2).

\section{DISCUSSION}

In this era of super-bugs, active surveillance of the multidrug resistant bacteria is extremely important for the provision of an efficient health-care (Gill et al., 2011). 
Table 2: Comparison of inhibition zone sizes $(\mathrm{mm})$ of EDTA alone with the difference in the zone sizes of Imipenem+EDTA (IPM+EDTA) and Imipenem (IPM) alone, for Pseudomonas isolates $(n=10)$.

\begin{tabular}{lllll}
\hline $\begin{array}{l}\text { Serial } \\
\text { No. }\end{array}$ & IPM & IPM+EDTA & $\begin{array}{l}\text { (IPM+EDTA) } \\
- \text { IPM }\end{array}$ & EDTA \\
\hline 1. & 8 & 29 & 21 & 10 \\
2. & 8 & 14 & 6 & 8 \\
3. & 13 & 27 & 14 & 10 \\
4. & 9 & 12 & 3 & 8 \\
5. & 12 & 29 & 17 & 10 \\
6. & 13 & 31 & 18 & 10 \\
7. & 8 & 30 & 22 & 11 \\
8. & 10 & 23 & 13 & 9 \\
9. & 9 & 29 & 20 & 9 \\
10. & 8 & 21 & 13 & 8 \\
\hline \multicolumn{5}{c}{ t-value $=3.115$} \\
\hline
\end{tabular}

MBLs are emerging as one of the most worrisome resistance mechanisms because they not only limit our treatment options but also their genes are carried on highly mobile elements, allowing their easy dissemination (Berges et al., 2007).

MBL-Etest has been reported to have a sensitivity and specificity of $100 \%$ for the detection of MBL producing GNRs (Walsh et al., 2002). In this study, we have compared CD test using imipenem and EDTA, which is an economical and simple method for MBL detection with the MBL-Etest. We found CD test as specific (100\%) as Etest but slightly less sensitive $(97.5 \%)$ than Etest. Our results are in concordance with many studies conducted in different parts of the world. In a study conducted in Belgium, Berges et al., (2007) found MBL-CD method, to have a sensitivity of $100 \%$ and specificity of $72.7 \%$ (Berges et al., 2007). Franklin et al., (2006), in their study, have reported CD test to have a sensitivity of $100 \%$ and specificity of $98 \%$ for MBL detection (Franklin et al., 2006). In another study conducted in Department of Laboratory Medicine, All India Institute of Medical Sciences, New Delhi, it was found that imipenem-EDTA combined disc test and imipenem-EDTA MBL Etest were equally effective for MBL detection (Bahrera et al., 2008). However many researchers have found results discordant to our findings. In a study conducted in Greece, 2008, imipenem-EDTA combined disc method and Etest method showed sensitivities of $80 \%$ and $70 \%$ respectively (Galani et al., 2008). Similarly Monoharam et al., (2010), in their study have reported a sensitivity and specificity of CD test of $87.8 \%$ and $84.4 \%$ respectively when compared to MBLEtest (Monoharan et al., 2010). This is quite low as compared to our results.

In the present study, among all carbapenem resistant isolates (on Kirby Bauer disc diffusion method), $77 \%$ of our isolates were found to be MBL producers and $21 \%$ negative for MBL production by both methods (CD Test and MBL-Etest). The reason for the carbapenem resistance in these Etest negative isolates on Kirby Bauer disc diffusion method might be other than MBL production, like decreased production of porins, efflux pumps or Amp C production (Quale et al., 2003; Urban et al., 2004).

Considering the possibility of false MBL detection due to changes in the permeability of the outer membrane, by using EDTA, among Pseudomonas isolates, we compared the inhibition zones of EDTA alone with the difference of inhibition zones of imipenem+EDTA and imipenem for our Pseudomonas isolates $(n=10)$. Our results were different from Ratkai et al., (2009) (t-value $=1.50, \mathrm{p}$-value $=0.17$ ) (Ratkai et al., 2009). On applying paired t-test we got a tvalue of 3.115 and $p$-value of 0.012 . This has ruled out the possibility of false MBL detection among our Pseudomonas isolates.

$\mathrm{CD}$ test is an economical and reliable test for MBL detection which can be employed for routine $\mathrm{MBL}$ detection in Gram negative bacilli. The technique is very easy, economical and can be incorporated into the routine testing of any busy microbiology laboratory. We recommend development of PCR for detection of MBL producing Gram negative bacilli in our set up and validation of $C D$ test and other simple, economical tests by their comparison with PCR.

\section{Conclusion}

Combined disc method using imipenem with EDTA is as effective a method as MBL-Etest for the detection of MBL producing Gram negative bacilli. However, given the cost constraints of MBL-Etest, a simple screening method like Combined disc imipenem/imipenem + EDTA method can be used.

\section{REFERENCES}

Arakawa, Y., Shibata, N., Shibibyama, K., Kurokawa, H., Yagi, T., Fujiwara, H. and Goto, M. (2000). Convenient test for screening of metallo- $\beta$-lactamase producing Gram- negative bacteria by using thiol compounds. Journal of Clinical Microbiology 38(1), 40-43.

Behera, B., Mathur, P., Das, A., Kapil, A. and Sharma, V. (2008). An evaluation of four different phenotypic techniques for detection of metallo- $\beta$-lactamase producing Pseudomonas aeruginosa. Indian Journal of Medical Microbiology 26(3), 233-237.

Berges, L., Rodriguez, H., Deplano, A. and Streneleus, M. J. (2007). Prospective evaluation of Imipenem/EDTA combined disc and Etest for detection of $\mathrm{MBL}$ producing Pseudomonas aeruginosa. Journal of Antimicrobial Chemotherapy 59(4), 812-813.

Clinical and Laboratory Standard Institute (2009). Performance standards for antimicrobial disk diffusion susceptibility tests. $19^{\text {th }}$ ed. Approved Standard, CLSI Document M100-S19. Vol. 29. CLSI, Wayne PA.

Etest MBL For in vitro detection of Metallo-BetaLactamases. Available Online: 
<http://www.abbiodisk.com/pdf/pi/75001546.PDF> [ Accessed on 27 June 2011]

Franklin, C., Liolios, L. and Peleg, A. Y. (2006). Phenotypic detection of carbapenem-susceptible metallo- $\beta$-lactamase-producing Gram-negative bacilli in the clinical laboratory. Journal of Clinical Microbiology 44(9), 3139-3144.

Galani, I., Rekatsina, P. D., Hatzaki, D., Souli, M. and Gamarellon, H. (2008). Evaluation of Different laboratory tests for the detection of metallo- $\beta$ lactamase production in Enterobacteriaceae. Journal of Antimicrobial Chemotherapy 61, 548-553.

Gill, M. M., Usman, J., Kaleem, F., Hassan, A., Khalid, A., Anjum, R. and Fahim, Q. (2011). Frequency and antibiogram of multi-drug resistant Pseudomonas aeruginosa. Journal of the College of Physicians and Surgeons Pakistan 21(9), 531-534.

Irfan, S., Zafar, A., Guhar, D., Ahsan, T. and Hasan, R. (2008). Metallo- $\beta$-lactamase-producing clinical isolates of Acinetobacter species and Pseudomonas aeruginosa from intensive care unit patients of tertiary care hospital. Indian Journal of Medical Microbiology 26(3), 243-245.

Kaleem, F., Usman, J., Hassan, A. and Khan, A. (2010). Frequency and susceptibility pattern of metallo-betalactamase producers in a hospital in Pakistan. Journal of Infectious Diseases in Developing Countries 4(12), 810-813.

Louis, B., Rice, Robert, A., Bonomo. (2007).(Arthur list not complete) Mechanism of resistance to antimicrobial agents. In: Murray, P. R. Baron, E. J. Landry, M. L. Jogensen, J. H. and Pfaller M. A. (eds.) Manual of Clinical Microbiology. $9^{\text {th }}$ edn. Washington DC, ASM Press. pp.1125-1128.

Marchiaro, P., Mussi, M. A., Ballerini, V., Pasteran, F., Viale, A. M., Vila, A. J. and Limansky, A. S. (2005). Sensitive EDTA based microbiological assays for detection of metallo- $\beta$-lactamases in non-fermenting Gram negative bacteria. Journal of Clinical Pathology 43(11), 5648-5652.

Monoharan, A., Chatterji, S., Matthai, D. et al. (2010). Detection and characterization of metallo beta lactamases producing Pseudomonas aeruginosa. Indian Journal of Medical Microbiology 28(3), 241244. (Arthur list not complete)

Quale, J., Bratu, S., Landman, D. and Heddurshetti, R. (2003). Molecular epidemiology and mechanisms of carbapenem resistance in Acinetobacter baumannii endemic in New York city. Clinical Infectious Diseases 37(2), 214-220.

Queenan, A. M. and Bush, K. (2007). Carbapenemases: the versatile B-Lactamases. Clinical Microbiology Reviews 20(3), 440-458.

Ratkai, C., Quinteira, S., Grosso, F., Monteiro, N., Nagy, E. and Peixe, L. (2009). Controlling for false positives: interpreting MBL Etest and MBL combined disc test for the detection of metallo-b-lactamases. Journal of Antimicrobial Chemotherapy 64(3), 657658.
Shobha, K. L., Lesaka, P. R., Sharma, M. K., Ramchandar, L. and Bairy, I. (2009). Metallo- $\beta-$ lactamase production among Pseudomonas species and Acinetobacter species in costal Karnataka. Journal of Clinical and Diagnostic Research 3, 17471753.

Urban., Carla., Rahal., James, J. B. (2004). )(Arthur list not complete). Mechanisms and detection of carbapenem resistance in Pseudomonas aeruginosa, Klebsiella pneumoniae, and Acinetobacter baumannii. Reviews in Merdical Microbiology 15(2), 63-72.

Walsh, T. R., Bolmstrom, A., Qwarnstrom, A. and Gales, A. (2002). Evaluation of a new Etest for detecting Metallo-_Lactamases in routine clinical testing. Journal of Clinical Microbiology 40(8), 27552759.

Yong, D., Lee, K., Yum, J. H., Shin, H. B., Rossolini, G. M., Chong, Y. (2002). Imipenem-EDTA disk method for differentiation of metallo- $\beta$-lactamases producing clinical isolates of Pseudomonas spp and Acinetobacter spp. Journal of Clinical Microbiology 40(10), 3798-801. 